\title{
Uber eine fundamentale Klasse morphogenetischer Regulationen.
}

\author{
Von \\ Hans Driesch.
}

Mit 2 Figuren im Text.

Eingegangen am 13. Mai 1908.

Wenn ein Keim, der ein Viertel, die Hälfte oder drei Viertel des Ganzen darstellt und durch Zerteilung dieses Ganzen in Richtung der polaren Symmetrieachse gewonnen ist, sich verkleinert ganz entwickelt, so sind nach meiner Auffassung bei diesem Geschehen vornehmlich nur sprimäre Regulationen beteiligt: die ontogenetischen Differenzierungsfaktoren selbst sind derart, daß sie verkleinerte Ganzentwicklung zulassen. Nur muß vorher die Intimstruktur des Keimbruchteiles ganz geworden sein: das allein ist eine ssekundäre oder echte Regulation*. Sekundäre Regulationen dieser Art beziehen sich also auf Umgestaltungen intimster organischer, besser morphogenetischer, Struktur. Sie bilden eine besondere Klasse echter organischer Regulationen.

Eine ganz andre, aber auch in sich einheitliche, Klasse von echten Regulationen wird dargestellt durch solche restitutiven Phänomene, welche ron unharmonisch zusammengesetzten Ausgängen ihren Ursprung nehmen. Jeder verstummelte erwachsene Organismus ist ein solcher unharmonisch zusammengesetzter Ausgang: seine Teile sind nicht in richtigem, "normalem "Verhältnis zneinander vorhanden; die Restitution, wenn sie eintritt, schafft wieder Harmonie der Zusammensetzung. Doch kann man auch von andern unharmonisch zusammengesetzten Ansgängen reden, nämlich von unharmonisch zusammengesetzten Keimteilen; ich habe diesen Begriff vor 3 Jahren gelegentlich einer mit solchen Keimteilchen sich befassenden Untersuchung ${ }^{1}$ ) geschaffen.

1) Dieses Arch. 19. 1905. S. 658. 
Ein unbarmonisch zusammengesetzter Keimteil liegt vor, wenn die verschiedenen, beim morphogenetischen Ablauf in Betracht kommenden Bestandteile des Keimes zwar alle vorhanden, aber in zueinander nicht passenden Quanten vorhanden sind. Echte $1 / 4^{-}, 1 / 2^{-}$und $3 / 4^{-}$ Keime sind also keine unharmonisch zusammengesetzten Keimteile.

Meine Untersuchung vom Jahre 1905 befaßte sich mit dem Studium des Mesenchyms an solchen Echinidenkeimen, welche verschieden große Bruchteile des animalen und des vegetativen Eiabschnittes besaßen; sie waren mit Hilfe des kalkfreien Seewassers ron Herbst hergestelit worden. Das wesentliche Resultat meiner Untersuchung war dieses: "Unharmonisch zusammengesetzte Keime, zu denen auch die mit nur animalen oder nur vegetativen Elementen zu rechnen sind, bilden in der überwiegenden Mehrzahl der Fälle eine Zahl von Mesenchymzellen, welche nicht der für sie giltigen Normzahl, d. h. nicht dem Bruchteil der normalerweise mesenchymbildenden Keimregion entspricht, welchen sie besitzen; sie liefern vielmehr je nach ihrer Zusammensetzung bald »zu viel «, bald »zu wenig « Mesenchymzellen, und zwar derart, daß die wirklich gelieferte Zahl dieser Elemente zum Keimwert der Objekte - d. h. zu der absoluten Größe des Bruchteils vom Keimganzen, welchen sie darstellen - annähernd in Proportion steht. Am extremsten zeigen dieses regulatorische Verhalten rein animale und rein regetative Keime. Neben dem Streben zur Erreichung der Proportion zum Keimwert geht eine bisweilen zum Durchbruch gelangende Tendenz zur Lieferung der Totalzahl des Mesenchyms einher $\ll 1$ ).

Des weiteren wurde festgestellt, daß die geschilderte Regulation in der Zahl der Mesenchymzellen gar nicht oder doch in nur höchst beschränktem Maße auf einer gesteigerten Häufigkeit der Teilung jener Zellen, also auf einer Reduction ihrer Größe beruht, daß sie vielmehr die Mesenchymmasse als Ganzes betrifft: jeweils in Proportion zum Keimwert wird ein bestimmter Massenbruchteil der vorbandenen Keimsubstanz zur mesenchymbildenden Region, mag diese Region mit Riucksicht auf das, was der vorhandene Keimbruchteil im Normalen an Mesenchym geliefert hätte, zu klein oder zu grob sein.

Daß auch die allgemeine Form der beginnenden Gastrula bei den morphogenetischen Ergebnissen unharmonisch zusammengesetzter embryonaler Ausgänge durchaus die verkleinert-normale war, wurde beiläufig festgestellt. Weiter ging jedoch die Untersuchung nicht. 
Ich teile nun zunächst die Ergebnisse nener Untersuchungen mit, in denen unharmonisch zusammengesetzte Keime bis zum Pluteus gezïchtet und dann auf ihre Proportionalität untersucht wurden.

Ich arbeitete diesmal mit dem Sechzehnzellenstadium membranlos gemachter Eier von Echinus. Mit Beginn der Sechzehnteilung kamen die Objekte in das kalkfreie Wasser, wurden etwas darin mit Hilfe der Pipette berumgeworfen, und dann wurden solche Stäcke ausgelesen, die eine nicht zueinander passende Anzahl von Micro-, Macro- und Mesomeren aufwiesen. "Zueinander passend , d. h., harmonisch zusammensetzend, würden natülich sein: $1 \mathrm{Mi}+1 \mathrm{Ma}+2 \mathrm{Me}(=1 / 4-\mathrm{Keim})$, oder $2 \mathrm{Mi}+2 \mathrm{Ma}+4 \mathrm{Me}(=1 / 2-\mathrm{Keim})$, oder $3 \mathrm{Mi}+3 \mathrm{Ma}+6 \mathrm{Me}(=3 / 4$-Keim); solche Bruchteile wurden also nicht verwendet. Man kann bei jedem Versuche nur auf sehr wenige geeignete Objekte rechnen. Ich trenne die Objekte in zwei Gruppen:

I. Der animale (Mesomeren-) Anteil des Keimes ist im Nachteil.

[Formel $n \cdot \mathbf{M i}+n \cdot \mathbf{M a}+(2 n-x)$ Me.]

Typus $2 \mathrm{Mi}+2 \mathrm{Ma}+2 \mathrm{Me}-3$ Objekte; 2 davon geben typische

Prismen mit dreigliedrigem Darm und Skelet, 1 gibt einen typischen Jungpluteus.

Typus $2 \mathrm{Mi}+2 \mathrm{Ma}+3 \mathrm{Me}-2$ Objekte; 1 typisches Prisma,

1 typischer Vollpluteus.

Typus $3 \mathrm{Mi}+3 \mathrm{Ma}+4 \mathrm{Me}-1$ Objekt; Vollpluteus.

Typus $3 \mathrm{Mi}+3 \mathrm{Ma}+5 \mathrm{Me}-1$ Objekt; Vollpluteus.

Typus $4 \mathrm{Mi}+4 \mathrm{Ma}+3 \mathrm{Me}-1$ Objekt; Vollpluteus.

Typus $4 \mathrm{Mi}+4 \mathrm{Ma}+4 \mathrm{Me}-3$ Objekte; alle typische Vollplutei.

Typus $4 \mathrm{Mi}+4 \mathrm{Ma}+5 \mathrm{Me}-1$ Objekt; Vollpluteus.

Typus $4 \mathrm{Mi}+4 \mathrm{Ma}+6 \mathrm{Me}-1$ Objekt; Vollpluteus, s. Fig. 1.

II. Der vegetative (Micro-, Macromeren-) Anteil des Keimes ist im Nachteil.

[Formel $(n-x)(\mathrm{Mi}+\mathrm{Ma})+n \mathrm{Me}$.]

Typus $1 \mathrm{Mi}+1 \mathrm{Ma}+5 \mathrm{Me}-1$ Objekt; typisches Prisma mit dreigliedrigem Darm und Skelet; 30 Mesenchymzellen (relativ zu viel für $1 \mathrm{Mi}+1 \mathrm{Ma} !)$.

Typus $1 \mathrm{Mi}+1 \mathrm{Ma}+6 \mathrm{Me}-2$ Objekte; 1 typisches Prisma mit 28 Mesenchymzellen (wieder relativ zu viel!); 1 typischer Jungpluteus, s. Fig. 2. 
Typus $1 \mathrm{Mi}+2 \mathrm{Ma}+4 \mathrm{Me}-1$ objekt; typiseher Jungpluteus. Typus $2 \mathrm{Mi}+2 \mathrm{Ma}+5 \mathrm{Me}-1$ Objekt; typischer Jungpluteus. Typus $2 \mathrm{Mi}+2 \mathrm{Ma}+6 \mathrm{Me}-1$ Objekt; typischer Jungpluteus. Typus $2 \mathrm{Mi}+2 \mathrm{Ma}+8 \mathrm{Me}-1$ Objekt; typiseher Jungpluteus.

Fig. 1.

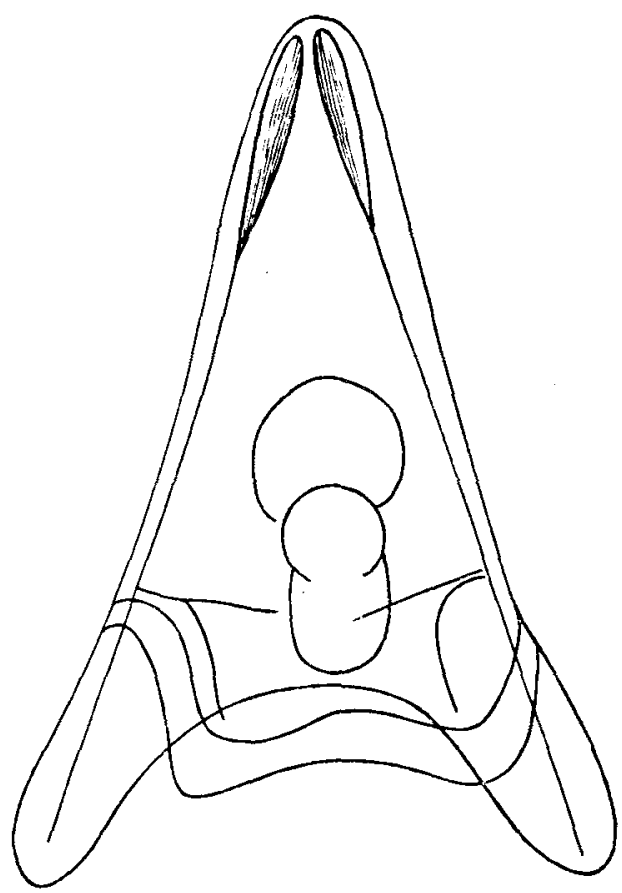

Echinus. Plateus aus Keimausgang rom Typus $4 \mathrm{Mi}+4 \mathrm{Ma}+6 \mathrm{Me}$.

Alle Larven waren durchaus normal-proportional ausgestaltet, trotz der fehlenden Harmonie in der Zusammensetzung ihres Ausganges.

In bezug auf die Geschwindigkeit der Entwicklung, anders gesagt in bezug auf die Erreichung des Zieles in gegebener

Fig. 2.

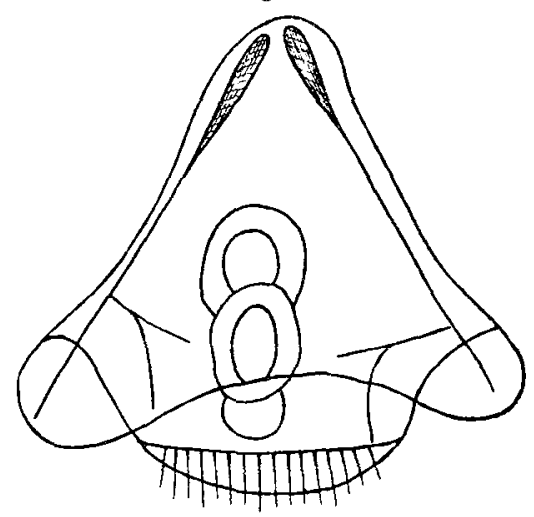

Echinus. Jungpluteus aus Keimausgang vom Typus $1 \mathrm{Mi}+1 \mathrm{Ma}+6 \mathrm{Me}$.

Zeit, sind die Objekte mit relativ zu viel vegetativem Keimmaterial (Gruppe I) etwas besser dran als die Gegengruppe. Aber das hat mit der harmonischen Ausbildung der Larren nichts zu tun. Hätte ich die Objekte der Gruppe II noch etwas länger gezüchtet, so hätten auch sie Plutei mit ganz langen Armen gegeben.

Unharmonisch zusammengesetzte Bruchteile des sechszehnzelligen Stadiums ron Echinus liefern also normal gebaute Larven.

Schon friber habe ich auf eine wichtige Entdeckung E. B. WILsons hingewiesen 1 ), welche uns Ähnliches wie meine eignen Versuche

1) Journ. exp. Zool. 1. 1904. p. 1. 
lehrt: Wird dem unreifen Ei von Dentalium ein beliebiger Teil seines animalen Abschnittes genommen, so gestaltet sich sein unharmonisch zusammengesetzter Rest so aus, daß er einen zum Keimwert an Größe passenden „Dottersack erhält und alles geht normal-verkleinert weiter; wird die Operation nach der Reifung gemacht, so wird aber der Dottersack »normal "-groß, d. h. für den Keimwert »zu groß

Ich selbst versuchte noch ein andres Experiment zur Frage nach der Entwicklung unharmonischer Ausgänge auszuführen. Conkur meint bekanntlich - wenigstens für den Ascidienkeim - daß rom Keimbläschen aus, bei dessen Auflösung zur Zeit der Reifung, gewisse der von ihm angenommenen formbildenden Stoffe geliefert würden. Mag die Berechtigung der Anuahme dahingestellt sein; auf alle Fälle wäre es von Interesse, an irgendwelchen Keimen das normale Verhältnis zwischen Keimbläschen und Plasmamenge zu stören und zu prïfen, was daraus folgt. In diesem Sinne trennte ich einer großen Anzahl unreifer Eier von Asterias einen Teil des Plasmas ab. Ein Restltat erzielte ich nicht, da das Material der vorgerückten Jahreszeit wegen inbrauchbar war und fast ausnahmslos mehrfach befruchtet wurde. Ich habe meinen Versuch hier mitgeteilt in der Hoffnung, daß sich viclleicht ein andrer an die Lösung der hier vorliegenden Aufgabe macht.

Wenn wir auch in Keimen, die abnorme Verhältnisse zwischen Chromatinsubstanz und Plasma aufweisen, embryonale Ausgänge von »unharmonischer Zusammensetzung" sehen wollen, so können wir bekanntlich sagen, daß solche Gebilde, nämlich parthenogenetische Keime einer-, Eibruchstucke mit vollständigem Kern anderseits, sich typisch entwickeln. Erstere bringen meist, aber nicht immer, erst durch Monasterbildung ihren Kern auf das richtige Maß ${ }^{1}$ ). Doch wissen wir nicht, ob hier wirklich unbarmonische Zusammensetzung in bezug auf für die eigentliche Differenzierung als solche wichtige Bestandteile des Keimes vorliegt.

Schon anläBlich meiner ersten Arbeit über den Gegenstand habe ich gesagt ${ }_{1}$ daß die Tatsache der normalen Entwieklung unharmonisch zusammengesetzter Ausgänge ohne weiteres den Schluß erlaubt, daß die in ihren Quanten nicht zueinander passenden Bestandteile des Ausganges jedenfalls nicht im eigentlich chemischen Sinne an der morphogenetischen Leistung beteiligt sind. Denn danu könnte es proportional-normale Morphogenese nur bei Wahrung der normalen Relation zwischen jenen Quanten geben. Es kommt aber nur darauf

!) Dieses Arch. 19. 1905. S. 648. 
an, daß jeder Bestandteil überhaupt anwesend ist. Eine naheliegende Annahme zur Erklärung der beobachteten Phänomene ist wohl diese, daß jene Bestandteile Protofermente oder Zymogene darstellen, welche je $\mathrm{n}$ ach Bed arf aktiviert werden: das würde erklären, daß jene Bestandteile überhaupt da sein müssen, ohne daß doch ihr absolutes oder relatives Quantum eine Rolle spielt. Zwar ein chemisches Faktum wäre solche Aktivierung vorgebildeter inaktivierter Bestandteile vom Ganzen aus durchans nicht, und so scheint es denn, als sähen wir in der Entwicklung unsrer unharmonisch zusammengesetzten Keime das vitale Problem zugleich in größter Einfachheit und in größter Klarheit vor uns.

Ich habe andernorts ${ }^{1}$ ) sehr eingehend die Gründe dargelegt, welche eine rein chemische Theorie der Morphogenese unannehmbar erscheinen lassen. Die Tatsache, daß von unharmonisch zusammengesetzten Ausgängen aus Harmonisches entstehen kann, ist mit unter diesen Gründen, obschon nicht als deren wesentlichster ${ }^{2}$ ). $\mathrm{Er}$ ist letzteres nicht, da wir eben doch nicht ganz sicher wissen, ob die "Bestandteile", die wir in ihrer unharmonischen Zusammensetzung im Keimausgang sehen, nun auch überhaupt morphogenetisch so. bedeutsam sind, wie wir meinen. Haben doch neuere Beobachtungen Garbowsisis ${ }^{3}$ ) den Boverischen Pigmentring des Strongylocentrotus, haben doch die Centrifugierungsversuche ${ }^{4}$ ) von Lyon und Morgan viele " organbildende Stoffe « andrer Keime als alles andre denn $\gg$ organbildend * erscheinen lassen. Beim Echinidenkeim wissen wir durch meine älteren Arbeiten ${ }^{5}$ ) immerbin so viel, daß animale und vegetative Keimhälften, je für sich genommen, sich wirklich recht verschiedenartig, obwohl gelegentlich ganz, entwickeln, und deshalb gewinnt gerade für diese Formen der Nachweis harmonischer Entwicklung unharmonischer Ausgänge mehr als bloß problematische Bedeutung.

Hat er aber diese größere Bedeutung - nun dann scheint mir auch, daß wir hier wirklich der vitalen Elementarleistung unmittelbar

1) Biol. Centr. 27. 1907. S. 60, und Gifford Lectures: The Science and Philosophy of the Organism. I. p. 134.

2) Der wesentlichste Grund für die Ablehnung jeder chemischen Theorie der Formbildung ist vielmehr die Tatsache, daß so viele chemisch gleich zusammengesetzte Bestandteile im Organismus in verschiedenen typischen Lagen sich befinden, und daß diese Bestandteile dazu noch jeweils, wie z. B. die Skeletteile, eine äußerst typisch individualisierte Form haben. Das vermöchte nur die Annahme einer *Maschine zu erklären, aber....

3) Bull. Acad. Cracovie. 1905. p. 509.

4) Dieses Arch. 23. 1907. S. 151, und 24. 1907. S. 147.

5 Dieses Arch. 10. 1900. S. 362, und 17. 1903. S. 41. 
152 Hans Driesch, Über eine fundamentale Klasse morphogenet. Regulationen.

gegenüberstehen: Das gegebene Stoffliche, mit dem die Morphogenese sich abfinden soll, paßt gar nicht in seinen Bestandteilen zueinander; gut, aber der vitale Faktor ist imstande, diese Bestandteile in solchen und nur in solchen Quanten zu verwerten, wie sie zueinander passen. Sie müssen nur überhaupt da sein.

Dieses ganze Räsonnement ist von der Theorie des harmonischäquipotentiellen Systems als solcher unabhängig. Es entsteht daher die Frage, ob es für sich genommen ein Beweis der Autonomie des Lebens ist. Das scheint mir nun freilich nicht der Fall zu sein, vielmehr läßt sich ein wahrer Beweis des Vitalismus ans der Tatsache der typischen Entwicklung unharmonisch zusammengesetzter Keime nur dann gewinnen, wenn man auch der Tatsache der harmonischen Äquipotentialität Rechnung trägt. Ein gegebener unharmonisch zusammengesetzter Keim $\mathrm{k} \ddot{o} \mathrm{n}$ te ja immerhin eine Maschinerie besitzen, welche der richtigen Aktivierung der hypothetischen Protofermente diente: erst die Tatsache, daß eben dieser unharmonisch zusammengesetzte Keim ohne Änderung des Resultats ganz ebenso gut in andrer Weise unharmonisch zusammengesetzt sein könnte, macht eine Maschinerie gedanklich unmöglich. Das aber ist die Argumentation der Theorie des harmonisch-äquipotentiellen Systems.

Die Möglichkeit, den Vitalismus zu beweisen, und die Tatsäghlichkeit vitalistischen Wirkens sind nun freilich zwei verschiedene Dinge. Erstere hängt an der Eigenart unsres Denkens, letztere am Gegebenen. In diesem Sinne können wir denn sagen, daß die Tatsache der harmonischen Entwicklung unharmonisch zusammengesetzter Ausgangspunkte, obschon sie an und für sich den Vitalismus nicht zu beweisen gestattet, sondern nur zeigt, daß diese Entwicklung nicht einfach chemisch von gegebenen Stoffquanten abhängt, daß diese Tatsache doch das vitale autonome Geschehen uns in besonders elementarer Form vor Augen führt.

Deshalb bildet die harmonische Entwicklung unharmonisch zusammengesetzter embryonaler Teile eine so wichtige und fundamentale Klasse aller Regulationen.

Wie nun eigentlich die Entelechie regulatorisch in das materielle Geschehen eingreift, wie im Besonderen sie in unserm Fall das richtige Quantum vorhandener Protofermente aktiviert, das auseinanderzusetzen ist hier nicht der Ort. Im zweiten Bande meiner * Gifford Lectures " habe ich versucht, eine ausgebante Theorie der Beziehungen $\mathrm{zwischen}$ Organischem und Unorganischem zu liefern.

Heidelberg, 9. Mai 1908. 